\title{
DRAKE: Dark matter Relic Abundance beyond Kinetic Equilibrium
}

\author{
Andrzej Hryczuk* \\ National Centre for Nuclear Research, \\ Pasteura 7, 02-093 Warsaw, Poland \\ E-mail: andrzej.hryczuk@ncbj.gov.pl
}

In the usual approach to the dark matter thermal relic abundance calculation an assumption of local thermal equilibrium is made. This proceedings presents a numerical precision tool DRAKE (drake.hepforge.org) that goes beyond this assumption by tracing not only the DM relic density, but also its velocity dispersion and full phase space distribution function. We review the general motivation for this approach and, for illustration, highlight two concrete classes of models where kinetic and chemical decoupling are intertwined in a way that can impact the value of the relic density by as much as an order of magnitude: dark matter annihilation via a narrow resonance and 'forbidden' annihilation to final states that are kinematically inaccessible at threshold.

*** The European Physical Society Conference on High Energy Physics (EPS-HEP2021), ***

*** 26-30 July $2021 * * *$

*** Online conference, jointly organized by Universität Hamburg and the research center DESY ***

${ }^{*}$ Speaker 


\section{Introduction}

The standard approach to calculate the thermal relic density [1], which can be recast in the form of a single Boltzmann equation for the dark matter (DM) number density, relies on the assumption of local thermal equilibrium of the DM component, i.e. that the DM is kept in kinetic equilibrium with the standard model (SM) heat bath during the whole chemical decoupling process. Public tools like DarkSUSY [2], MadDM [3], micrOMEGAs [4] and SuperISOrelic [5] rely on this assumption as well. There exist however various well-motivated scenarios where this condition is not satisfied and where the standard approach is not justified.

Here we report on introducing a new public code, DRAKE [6] (Dark matter Relic Abundance beyond Kinetic Equilibrium) ${ }^{1}$, which for the case of annihilating DM implements: $i$ ) coupled equations for the number density and higher moments of the DM phase space distribution and ii) the full Boltzmann equation directly at the phase space level. Therefore, DRAKE provides a tool to predict the relic density also in situations where the underlying assumptions of the traditional approach cannot be justified.

DRAKE is written fully in Wolfram Language and can be used either in Mathematica notebook environment or externally, without loosing any functionality, as a script making use of the Wolfram Engine.

\section{Scope and code design}

\subsection{Full Boltzmann equation}

The evolution of the DM phase-space density $f_{\mathcal{X}}(t, p)$ during the freeze-out process is determined by what we refer to as the full Boltzmann equation (fBE):

$$
E\left(\partial_{t}-H p \partial_{p}\right) f_{\mathcal{X}}=C_{\mathrm{ann}}\left[f_{\mathcal{\chi}}\right]+C_{\mathrm{el}}\left[f_{\mathcal{\chi}}\right]
$$

where the annihilation part, under the assumptions of $\mathrm{CP}$ invariance and $f_{\chi} \ll 1$, can be reduced to only two integrations:

$$
C_{\mathrm{ann}}=g_{\chi} E \int \frac{d^{3} \tilde{p}}{(2 \pi)^{3}} v_{\mathrm{M}} \sigma_{\bar{\chi} \chi} \rightarrow \bar{f} f\left[f_{\mathcal{X}, \mathrm{eq}}(E) f_{\mathcal{X}, \mathrm{eq}}(\tilde{E})-f_{\mathcal{X}}(E) f_{\mathcal{X}}(\tilde{E})\right],
$$

while the collision term for elastic scattering processes can be given within the Fokker-Planck type approximation $[7,8]$ including some of the leading relativistic corrections $[9,10]$, resulting in:

$$
C_{\mathrm{el}} \simeq C_{\mathrm{FP}}=\frac{E}{2} \gamma(T)\left[T E \partial_{p}^{2}+\left(2 T \frac{E}{p}+p+T \frac{p}{E}\right) \partial_{p}+3\right] f_{\chi} .
$$

The momentum transfer rate $\gamma(T)$ is given by

$$
\gamma=\frac{1}{3 g_{\chi} m_{\chi} T} \int \frac{\mathrm{d}^{3} k}{(2 \pi)^{3}} g^{ \pm}(\omega)\left[1 \mp g^{ \pm}(\omega)\right] \int_{-4 k_{\mathrm{cm}}^{2}}^{0} \mathrm{~d} t(-t) \frac{\mathrm{d} \sigma}{\mathrm{d} t} v,
$$

\footnotetext{
${ }^{1}$ Publicly available at drake.hepforge.org.
} 
where $k_{\mathrm{cm}}^{2} \equiv m_{\chi}^{2} k^{2} /\left(m_{\chi}^{2}+2 \omega m_{\chi}+m_{f}^{2}\right)$ and the scattering amplitude, $(\mathrm{d} \sigma / \mathrm{d} t) v \equiv|\mathcal{M}|_{\chi f \leftrightarrow \chi f}^{2} /\left(64 \pi k \omega m_{\chi}^{2}\right)$, is evaluated at $s \simeq m_{\chi}^{2}+2 \omega m_{\chi}+m_{f}^{2}$ and implicitly summed over all heat bath particles $f$, and final and initial state internal degrees of freedom.

The Fokker-Planck approximation is very well justified when the mass of the scattering partner is much smaller than the DM mass, such that the momentum transfer is small. If this is not the case one has to resort to the full scattering term. This possibility is also introduced in DRAKE, though is significantly more CPU expensive.

\subsection{Fluid equations}

As an alternative to Eq. (1) one can consider only its $p$ moments. A convenient parameterization for this is the DM velocity dispersion or 'temperature', $T_{\chi} \equiv g_{\chi} /\left(3 n_{\chi}\right) \int d^{3} p(2 \pi)^{-3}\left(p^{2} / E\right) f_{\chi}$, and one way of closing the Boltzmann hierarchy at this level is to assume $f_{\mathcal{X}}=\exp \left[(\mu-E) / T_{\chi}\right]$. Using Eq. (3), and assuming entropy conservation, this results in what we refer to as the coupled Boltzmann equations (cBE):

$$
\begin{aligned}
\frac{Y^{\prime}}{Y}= & \frac{s Y}{x \tilde{H}}\left[\frac{Y_{\mathrm{eq}}^{2}}{Y^{2}}\langle\sigma v\rangle_{T}-\langle\sigma v\rangle_{T_{\chi}}\right], \\
\frac{y^{\prime}}{y}= & \frac{1}{x \tilde{H}}\left\langle C_{\mathrm{el}}\right\rangle_{2}+\frac{s Y}{x \tilde{H}}\left[\langle\sigma v\rangle_{T_{\chi}}-\langle\sigma v\rangle_{2, T_{\chi}}\right] \\
& +\frac{s Y}{x \tilde{H}} \frac{Y_{\mathrm{eq}}^{2}}{Y^{2}}\left[\frac{y_{\mathrm{eq}}}{y}\langle\sigma v\rangle_{2, T}-\langle\sigma v\rangle_{T}\right]+2(1-w) \frac{H}{x \tilde{H}},
\end{aligned}
$$

where $w\left(T_{\chi}\right) \equiv 1-\left\langle p^{4} / E^{3}\right\rangle_{T_{\chi}} /\left(6 T_{\chi}\right)$ characterises the deviation from the deeply non-relativistic case $(w=1)$. In order to arrive at this form, we followed the standard convention of introducing dimensionless variables $Y(x) \equiv n / s, y(x) \equiv m_{\chi} T_{\chi} s^{-2 / 3}$ and $x \equiv m_{\chi} / T$, where $s$ is the entropy density. The thermal average $\langle\sigma v\rangle_{2, T}$ is a variant of the commonly used thermal average $\langle\sigma v\rangle_{T}$, and is given in Ref. [10]. We also used

$$
\left\langle C_{\mathrm{el}}\right\rangle_{2} \equiv \frac{g_{\chi}}{3 n T_{\chi}} \int \frac{\mathrm{d}^{3} p}{(2 \pi)^{3}} \frac{p^{2}}{E^{2}} C_{\mathrm{el}},
$$

which in the Fokker-Planck approximation of Eq. (3) simplifies to $\left\langle C_{\mathrm{el}}\right\rangle_{2} \rightarrow \gamma(T) w\left(T_{\mathcal{X}}\right)\left[\left(y_{\mathrm{eq}} / y\right)-1\right]$. and $\tilde{H} \equiv H /\left[1+(1 / 3) d\left(\log g_{\text {eff }}^{s}\right) / d(\log T)\right]$, with $g_{\text {eff }}^{s}$ denoting the entropy degrees of freedom of the background plasma.

\subsection{Code design}

DRAKE provides routines to solve the individual Boltzmann equations ( $\mathbf{C B E}$ and $\mathbf{f B E}$ introduced above and also the standard approach we call $\mathbf{n B E}$ ), as well as a set of preparatory routines that compute quantities required by the solvers, e.g., averages of the annihilation cross-section. This structure is designed to perform relic abundance computations in a flexible manner and to reduce the required model input to fairly simple expressions.

These solvers are adaptive and implicit where the partial differential Eq. (1) for the fBE solver has been mapped to a set of ordinary differential equations by the method of lines. Each of the 
solvers calculates the relic abundance $\Omega_{\chi} h^{2}$ and the evolution of $Y(x)$. cBE and fBE also give the evolution of $y(x)$, and the latter additionally provides results for the phase-space density $f_{\mathcal{X}}(x, p)$.

The annihilation cross-section averages needed in the Boltzmann equations are computed by the supplied routines. The only input required from the user is the DM annihilation cross-section in the form of $\sigma v_{\text {lab }}$ as a function of the Mandelstam variable $s$. The routines then adopt to adjustable accuracy settings and the DM model.

For the $\mathrm{cBE}$ and $\mathrm{fBE}$ routines also the scattering operator $C_{\mathrm{el}}$ needs to be calculated. If Eq. (3) is used for the scattering operator, then the rate $\gamma(x)$ in Eq. (4) (or only its the model-dependent part, the $t$-average of the scattering amplitude $|\mathcal{M}|_{\chi f \leftrightarrow \chi f}^{2}$ ) should be provided by the user.

The first code release came with five pre-implemented DM model setups. These are the Scalar Singlet DM, resonant annihilation, Sommerfeld-enhanced annihilation, sub-threshold annihilation and WIMP-like models. It also contains example notebook and script for standard usage, as well as a script for adding new models.

\section{Example physics scenarios}

To showcase the application of DRAKE here we briefly present two selected examples. More extended discussion and details can be found in [6].

\subsection{Resonant annihilation}

For the first example, let's take a scenario where the annihilation cross-section exhibits a strong velocity dependence coming from a resonance. For concreteness we choose a generic vector mediator $A^{\mu}$ inducing an $s$-channel resonance. A minimal version of such a scenario is described by the interaction Lagrangian

$$
\mathcal{L} \supset-g_{\chi} \bar{\chi} \gamma^{\mu} \chi A_{\mu}-g_{f} \bar{f} \gamma^{\mu} f A_{\mu}
$$

leading to a Dirac fermion DM particle $\chi$ annihilating into heat bath fermions $f$. The model can be described by a set of 5 parameters: $m_{\chi}$ is the DM mass, $r \equiv m_{f} / m_{\chi}, \tilde{\gamma} \equiv \Gamma_{A} / m_{A}$ is a dimensionless measure of the total decay width of $A^{\mu}, \rho \equiv \sqrt{g_{\chi} g_{f}}$ is the combination of the relevant coupling constants and $\delta \equiv\left(2 m_{\chi} / m_{A}\right)^{2}-1$ gives the deviation from the exact resonance position.

In Fig. 1 we show the impact on the relic abundance when varying the resonance width $\tilde{\gamma}$ and keeping the mass ratio $r$ fixed. Looking at the contours one can see that the size of the effect can exceed an order of magnitude. For most of the parameter space a smaller width leads to a larger deviations from the standard computation. It is interesting to note that even for relatively large widths, like that of the SM Z-boson, our improved prediction of the DM abundance can deviate at a level well larger than $\sim 1 \%$ in $\Omega_{\mathrm{DM}} h^{2}$.

Finally we remark that the narrower the resonance, the more momentum-selective is the annihilation. This can lead to the distribution function that strongly differs from thermal one and the $\mathbf{f B E}$ approach implemented in DRAKE can accurately resolve such non-obvious phase-space evolution even for extremely narrow resonances. 


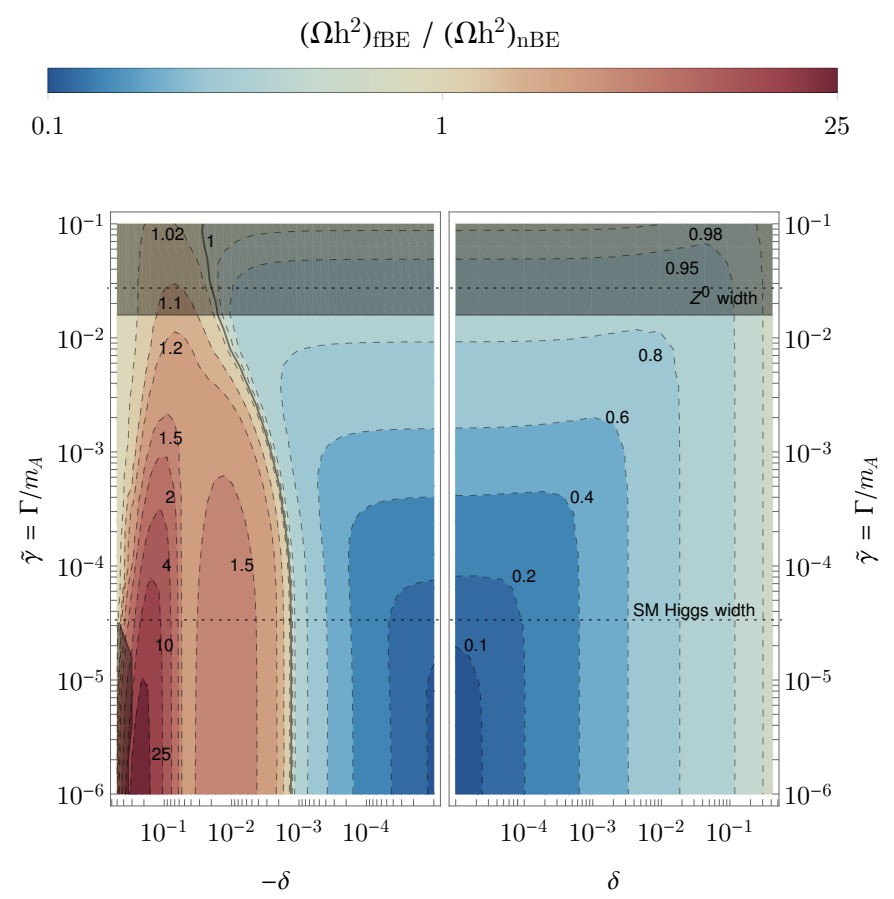

Figure 1: The effect for the relic density for an example resonance model. Contour lines give $\left(\Omega_{\chi} h^{2}\right)_{\mathrm{fBE}} /\left(\Omega_{\chi} h^{2}\right)_{\mathrm{nBE}}$. The parameters are $m_{\chi}=1 \mathrm{GeV}$ and $r=0.3$ while couplings are fixed such that $2\left(\Omega_{\chi} h^{2}\right)_{\mathrm{nBE}}=\Omega_{\mathrm{DM}} h^{2}$, and $\delta=\left(2 m_{\chi} / m_{A}\right)^{2}-1$. Red (blue) colors indicate regions where the early kinetic decoupling effect overall increases (decreases) the relic abundance compared to the usual treatment. The gray shaded areas on the edges of the plot show regions where the coupling value satisfying the relic density condition violates perturbativity.

\subsection{Sub-threshold annihilation}

As a second example take a situation where the annihilation process that determines the relic density is kinematically not accessible at rest, sometimes referred to as 'forbidden' DM [11]. For concreteness, consider an interaction Lagrangian

$$
\mathcal{L} \supset-\frac{\lambda}{4} \phi_{1}^{2} \phi_{2}^{2}+y_{f} \phi_{2} \bar{f} f
$$

where the scalar $\phi_{1}$ plays the role of the DM particle. At tree level it interacts only with the scalar $\phi_{2}$. We assume that $\phi_{2}$ is close in mass to $\phi_{1}$, i.e., $r \equiv m_{2} / m_{1} \sim 1$, and is in thermal contact with the heat bath fermions $f$.

The results for such scenario are given in Fig. 2. In the considered parameter region, the momentum transfer rate, Eq. (3), is then given by

$$
\gamma(x) \approx m_{1} \frac{\lambda^{2}}{6 \pi^{3}} \frac{r^{2}}{(1+r)^{4}} x^{-2} e^{-r x} .
$$

It follows that the kinetic decoupling can happen very early in this model, around the time of chemical decoupling. However, the approximate scattering collision term in Eq. (3), including the momentum transfer rate, relies on the assumption of small momentum transfer compared to the 


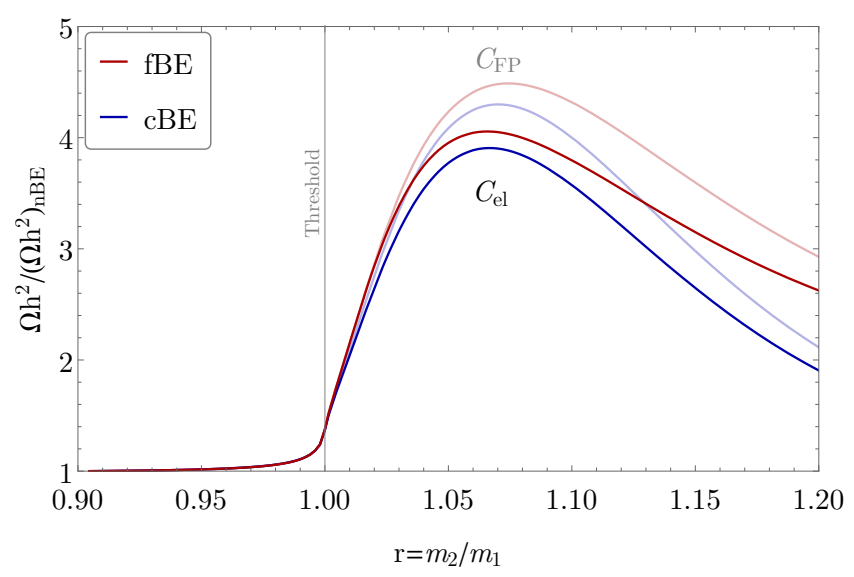

Figure 2: The size of the effect on the relic abundance for the sub-threshold annihilation example, for DM mass of $m_{1}=100 \mathrm{GeV}$. The value of the $\phi_{1}-\phi_{2}$ coupling is determined by the requirement $\left(\Omega_{\chi} h^{2}\right)_{\mathrm{nBE}}=$ $\Omega_{\mathrm{DM}} h^{2}$. Solid red (blue) lines correspond to results from the fBE $(\mathbf{c B E})$ treatment based on the full scattering term, while the results using the Fokker-Planck approximation are plotted with lighter shading.

average DM momentum. This is typically not the case for scattering partners that have a mass similar to the DM particle.

In [6] we demonstrate that in this case it is possible to instead implement the scattering collision term $C_{\mathrm{el}}$ without this assumption. This allows us to contrast our results to those based on the approximate scattering term $C_{\mathrm{FP}}$ (relying on small momentum transfer).

In Fig. 2 we plot the relic density that results from the $\mathbf{f B E}$ and $\mathbf{c B E}$ approaches, with different implementations of the scattering terms, relative to that from the $\mathbf{n B E}$ approach. Here, for each value of $r$, the coupling $\lambda$ is fixed by the requirement that the standard $\mathbf{n B E}$ prediction matches the observed abundance. As is clearly visible, early kinetic decoupling can have a significant impact on the relic abundance also for this threshold example, at least for $r \gtrsim 1$.

\section{Summary}

We have presented a new public tool, DRAKE, that calculates the DM relic density beyond the usual assumption that kinetic equilibrium is maintained during the freeze-out. The code offers two alternative schemes to calculate the relic density that take into account the effects of kinetic decoupling during the chemical freeze-out process, including a full calculation at the phase-space level.

This is clearly important for global fits that include parameter regions in their scans where the interplay between kinetic and chemical equilibrium cannot be neglected, but can turn out to be relevant also in various model-building considerations.

Last but not least let us stress that even though the main goal of DRAKE is to calculate the relic density, its output is by no means restricted to this single result. Indeed, DRAKE allows to compute the full time evolution of the DM phase-space density, or its lowest moments, which may be used and further connected to other late-time observables, e.g. a non-thermal velocity distribution that affects the matter power spectrum of density perturbations (see e.g. $[12,13]$ ). 


\section{Acknowledgements}

A.H. is supported by the National Science Centre, Poland, grant No. 2018/31/D/ST2/00813.

\section{References}

[1] P. Gondolo and G. Gelmini, Cosmic abundances of stable particles: Improved analysis, Nucl. Phys. B 360 (1991) 145.

[2] T. Bringmann, J. Edsjö, P. Gondolo, P. Ullio and L. Bergström, DarkSUSY 6 : An Advanced Tool to Compute Dark Matter Properties Numerically, JCAP 07 (2018) 033 [1802 . 03399$].$

[3] F. Ambrogi, C. Arina, M. Backovic, J. Heisig, F. Maltoni, L. Mantani et al., MadDM v.3.0: a Comprehensive Tool for Dark Matter Studies, Phys. Dark Univ. 24 (2019) 100249 [1804.00044].

[4] G. Belanger, F. Boudjema, A. Pukhov and A. Semenov, MicrOMEGAs 2.0: A Program to calculate the relic density of dark matter in a generic model, Comput. Phys. Commun. 176 (2007) 367 [hep-ph/0607059].

[5] A. Arbey and F. Mahmoudi, SuperIso Relic: A Program for calculating relic density and flavor physics observables in Supersymmetry, Comput. Phys. Commun. 181 (2010) 1277 [0906.0369].

[6] T. Binder, T. Bringmann, M. Gustafsson and A. Hryczuk, DRAKE: Dark matter Relic Abundance beyond Kinetic Equilibrium, Eur. Phys. J. C 81 (2021) 577 [2103.01944].

[7] E. Bertschinger, The Effects of Cold Dark Matter Decoupling and Pair Annihilation on Cosmological Perturbations, Phys. Rev. D 74 (2006) 063509 [astro-ph/0607319].

[8] T. Bringmann and S. Hofmann, Thermal decoupling of WIMPs from first principles, JCAP 0704 (2007) 016 [hep-ph/0612238].

[9] T. Binder, L. Covi, A. Kamada, H. Murayama, T. Takahashi and N. Yoshida, Matter Power Spectrum in Hidden Neutrino Interacting Dark Matter Models: A Closer Look at the Collision Term, JCAP 1611 (2016) 043 [1602.07624].

[10] T. Binder, T. Bringmann, M. Gustafsson and A. Hryczuk, Early kinetic decoupling of dark matter: when the standard way of calculating the thermal relic density fails, Phys. Rev. D 96 (2017) 115010 [1706.07433].

[11] R.T. D’Agnolo and J.T. Ruderman, Light Dark Matter from Forbidden Channels, Phys. Rev. Lett. 115 (2015) 061301 [1505.07107].

[12] D. Boyanovsky and J. Wu, Small scale aspects of warm dark matter : power spectra and acoustic oscillations, Phys. Rev. D 83 (2011) 043524 [1008. 0992].

[13] P. Hager and A. Kassiteridis, Phase-space Distribution Functions of Feebly Particles and Their Signatures, 2009.11308. 